\title{
Autoridad burlesca y modernidad en el teatro breve barroco ${ }^{7}$

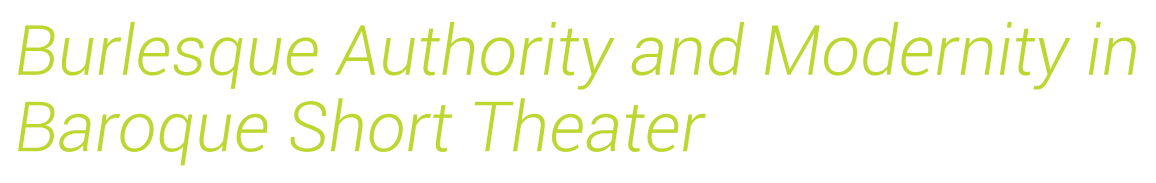

\section{Antonio Guijarro-Donadiós}

Department of Literatures, Cultures and Languages

365 Fairfield Way/Oak Hall 207B

College of Liberal Arts and Sciences

University of Connecticut

Storrs, CT 06269-1057, EE.UU.

antonio.guijarro@uconn.edu

[Hipogrifo, (issn: 2328-1308), 1.1, 2013, pp. 201-210]

Recibido: 14-02-2013 / Aceptado: 14-03-2013

DOI: http://dx.doi.org/10.13035/H.2013.01.01.14

Resumen. Este artículo estudia la comicidad provocada por las nuevas formas de ocio y consumo en el teatro breve barroco mediante el aprovechamiento dramatúrgico del Alcalde entremesil inserto en el ámbito urbano. Se analizan diversos entremeses donde la comercialización y uso de nuevos productos y hábitos sirven de motivo principal para burlarse de la figura de autoridad rural en la emergente sociedad de consumo del siglo XVII.

Palabras clave. Alcalde, entremés, comicidad, cultura material.

Abstract. The purpose of this article is to study how the trends of diversion and consumption determined the comic elements of the short plays through the analysis of the comic Mayor within the urban space. I focus on several interludes where commerce and the use of new products and habits are the principal means of making fun of the rural authority figure in the emerging new society of consumers in the 17 th century.

Keywords. Mayor, Interlude, Humor, Material Culture.

1. Este trabajo ha sido posible gracias a la Beca de Investigación del Departamento de Humanidades de la Universidad de Connecticut (UCHI). 
La administración de justicia civil en la vida cotidiana del siglo XVII estaba formada de diversas autoridades que ejercían su poder de acuerdo con una estricta jerarquía. En la Corte, asistiendo al Rey, estaba el Consejo Real. A escala regional, las Audiencias y Chancillerías se veían pobladas de oidores, jueces, procuradores, fiscales y corregidores ${ }^{2}$. Más cercanos a la gente común y corriente estaban los escalones inferiores de la justicia, esto es, pertenecientes a la administración municipal, compuesta de escribanos, alguaciles, regidores y sobre todo, alcaldes, de cuyas 'alcaldadas' o necedades se nutrían loas, jácaras, entremeses, bailes y mojigangas que conforman el denominado teatro breve. El alcalde, heredero del pastor bobo de los introitos de Torres Naharro y del simple de los pasos de Rueda queda así configurado como un personaje o tipo característico, cuya aparición en escena debía reconocerse en seguida tanto por su vestuario como por su forma de expresión. De hecho, aparece representado en múltiples ocasiones con vara de alcalde, pero también con la caperuza y el sayo que evocan el rústico ${ }^{3}$. En la continuación - Segunda parte de la vida del pícaro Guzmán de Alfarache de Juan Martí Luján de Sayavedra aparecía definido en el octavo capítulo de la siguiente manera: «Lo del simple que usan en España es bueno sin perjuicio, porque causa risa, empezando muchas sentencias y acabando ninguna, haciendo mil precisiones muy graciosas, y es un personaje que suele deleitar más al vulgo que cuantos salen a las comedias, en razón de que en él cabe ignorancia y malicia, y lascivia rústica y grosera» ${ }^{4}$.

Este alcalde, símbolo de la autoridad burlesca, aparece como un personaje de procedencia rural cuya locura, ignorancia y despropósitos en su afán de velar por el orden público provocarán la risa y los aplausos en los corrales formados mayoritariamente por una audiencia urbana.

La mayor parte de las piezas breves donde aparece este alcalde villano tienen lugar en el espacio de ficción de la aldea, de sobra son conocidas para el lector y estudioso piezas del tipo La elección de los alcaldes de Daganzo, El retablo de las maravillas, El alcalde de Burguillos o la serie de entremeses benaventina de Los alcaldes encontrados. Obras estas, donde son lugares comunes la pugna entre alcalde y escribano, el marido cornudo, el orgullo de su linaje de cristiano viejo y su profunda religiosidad, aun a costa de su ignorancia y analfabetismo. Valores pertenecientes a un pasado no muy lejano que no tienen cabida en el vertiginoso crecimiento de la ciudad. Aún más interesante resulta el aprovechamiento dramatúrgico de esta figura de autoridad inserta en el ámbito urbano en relación con la emergente sociedad de consumo. Cabe destacar que el público esencial que ríe estas 'alcaldadas' habita las ciudades, espacios que, con sus mecanismos de extrañamiento e integración, de consumo masificado y de espacio para la singularidad se afianzan como escenario privilegiado de la cultura y de la vida en general en el Barroco ${ }^{5}$.

2. Fernández Álvarez, 1983, pp. 265-268.

3. Para una aproximación general al motivo rústico en la comedia española, véase el clásico estudio de Nöel Salomon, Lo villano en el teatro del Siglo de Oro. Para este trabajo, me fueron valiosas las páginas correspondientes a la Primera Parte: El villano cómico (20-149); especialmente el Capítulo IV: Los Alcaldes (91-122)

4. Cotarelo, 1904, p. 440.

5. Ruiz Pérez, 2010, pp. 11-13

HIPOGRIFO, 1.1, 2013 (pp. 201-210) 
Con un mundo rural en continuo despoblamiento por el doble impulso de la crisis económica y el atractivo de la vida en la ciudad, que aparecía como más descansada que la dura realidad del campo, Madrid, Sevilla, Cádiz, Barcelona y Valencia aumentan demográficamente y su actividad comercial las convierte en núcleos urbanos mercantiles claves durante el siglo XVII. La relevancia de estas ciudades se produce, en parte, gracias a su intercambio de ideas y mercancías con el norte de Europa, el Nuevo Mundo y el Mediterráneo. Esta permuta hace que la literatura del periodo se vea inundada de nuevos objetos que influyen en los hábitos sociales originando un proceso de transculturización a través de viajes, guerras, movimientos migratorios, colonización y sobre todo, mediante el comercio.

La topografía de estos ámbitos urbanos adquiere un valor simbólico y los espacios se resemantizan cargándose de significación. Los ambientes principales (plazas, mercados, mentideros...) están conectados con los objetos de consumo allí adquiridos, (tabaco, chocolate, joyas, tejidos...) con el material humano que los ocupa (pedigüeñas, tahúres, gitanos...) y por último, con los asuntos tratados: la expulsión de los moriscos, el lujo y la novedad, el mundo del hampa o la moda. Todo queda plasmado en estas piezas breves cuyo resultado es la construcción narrativa de la ciudad con todo aquello que lo puebla y la articulación de la urbe como elemento clave del texto.

Esta lectura del fenómeno urbano en relación con el comportamiento humano y del objeto como articulador cultural y no sólo como mero producto ha sido denominado por la crítica reciente como Nuevo Materialismo o Materialismo Culturalb. Esta propuesta se centra en la cultura material del periodo y sus implicaciones sociales, económicas y políticas ofreciendo interpretaciones de la cultura barroca apenas antes consideradas. En este contexto se sitúa la propuesta de este trabajo que, ampliando el discurso a otras disciplinas de las humanidades, estudia la resonancia social de elementos cotidianos aparentemente corrientes o prescindibles dentro del entorno urbano. Con ello, atiendo no sólo a los aspectos materiales de la vida cotidiana del ciudadano barroco, sino también, como Enrique García SantoTomás ha subrayado, «a las reverberaciones simbólicas de determinados objetos sólidamente imbricados en su cotidianeidad»? ${ }^{7}$. El teatro cómico breve se presta a este análisis, puesto que las formas de ocio y consumo determinan, en gran parte, su comicidad, reflejando los placeres, las ansiedades y miedos que se asocian a los nuevos hábitos en el ámbito urbano dentro de la incipiente modernidad que atraviesa la sociedad del XVII.

Un ejemplo que explica esta simbiosis aparece en el Entremés de las lenguas atribuido a Jerónimo de Cáncer y Velasco, publicado en 1691 en Madrid dentro de la Floresta de Entremeses y rasgos del ocio. La pieza comienza con la llegada de un alcalde rural, orgulloso de sus valores de linaje y limpieza de sangre, a un Madrid cosmopolita en busca de un baile para las fiestas de su aldea. La primera impresión que le produce muestra su total desconcierto «pues a Madrid venía / y me hallo 
dentro de Babilonia» ${ }^{8}$. En la búsqueda de la danza, interroga y se mofa, como el título indica, de los dialectos de valencianos, gallegos, italianos, irlandeses y portugueses que salen a su encuentro. A lo largo de esta Sierra Morena madrileña, el alcalde demanda la presencia de negros y tras oír mencionar su casta, sale uno ofreciéndose, no a bailar, como espera el alcalde, sino sorprendentemente a moler el chocolate y a aderezarlo con insólitos ingredientes; dejando así constancia de una nueva profesión urbana, la de destilador, que ejerce un comerciante cuyo oficio y etnia es marginada:

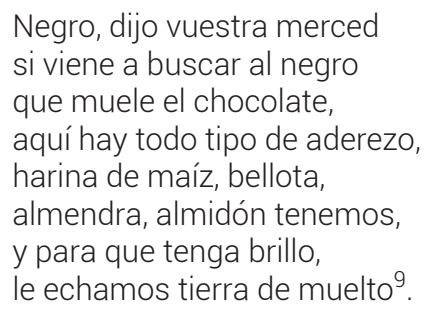

Junto a la comicidad del dialecto del hombre negro, el abusivo hábito de beber chocolate mejicano que altera el cuerpo humano causando hinchazón del estómago y flatulencias se conforma dentro de las características típicas del humor carnavalesco propio de estas piezas. Es interesante notar cómo la demanda de chocolate provocó también que los destiladores añadieran en su confección todo tipo de rellenos para aumentar la especulación, como harina de maíz, bellotas e incluso tierra de muertos como aparece continuamente en la prensa de la época. En los Avisos de Jerónimo de Barrionuevo leemos un suceso de 1657, donde un fraile descalzo que predicó en San Gil, llegó al Consejo Real relatando que «había llegado a sus pies un penitente que mezclaba el chocolate con tierra de difuntos, que lo engrasaba mucho y hacía muy bueno, y que con esto lo vendía a muy subido precio» ${ }^{10}$.

En el caso que nos ocupa, no sólo aparece estereotipado el negro de malos hábitos higiénicos, sino la incorporación de un elemento corrupto añadido a un ya de por sí producto exótico es un síntoma de los tiempos que corren: una bebida adulterada que consume una sociedad carente de valores en una ciudad enferma. El chocolate formará parte de la cultura urbana y pronto aparecerán tratados culinarios que incluyen discusiones médicas, crítica social y prevención de males con la observación de costumbres. Antonio de León Pinelo indicaba en su obra Cuestión moral si el chocolate quebranta el ayuno eclesiástico cómo «ahora se vende en cajas, panes o tabletas y por aumentar la pasta y hacer que rinda mucho con poco cacao, que es acá el material costoso, le mezclan almidón y harinas de diferentes granos y frutos ${ }^{1}{ }^{1}$. Indicativo de este tiempo es también la propuesta de Santiago Valverde Turices, quien en su obra Un discurso del chocolate, publicado en 1624,

8. Cáncer y Velasco, Entremés de las lenguas, p. 37.

9. Cáncer y Velasco, Entremés de las lenguas, p. 42.

10. Barrionuevo, Avisos de D. Jerónimo de Barrionuevo, vol. III, p. 214.

11. León Pinelo, Questión moral si el chocolate quebranta el ayuno eclesiástico, fol. 89r. 
establece una conexión entre la pimienta como cualidad del buen chocolate y la lujuria como desviación probable por su consumo debido a las propiedades aromáticas que seducían a las damas ociosas y con posibles de la corte madrileña del XVII. Como vemos, la comercialización de materias primas importadas del Nuevo Mundo, se establecen como marcas de relación social, como indicadoras de un determinado capital simbólico ${ }^{12}$ y como creadoras, en consecuencia, de una serie de prácticas sociales ${ }^{13}$, donde pueden observarse las estructuras de clase o las relaciones de poder desde un objeto o un suceso en particular ${ }^{14}$.

Las visitas al Camposanto debían ser numerosas durante el tiempo que nos ocupa a tenor de lo que leemos en estas piezas breves. Además de la utilidad de la 'tierra de muertos' para adulterar el chocolate, y junto al ya conocido macabro relleno de los pasteles de carne, los cabellos de los difuntos ofrecían un uso práctico en la elaboración de postizos y pelucas. La moda se convierte así en una de las preocupaciones del urbanita, bien como procedimiento de ostentación, bien como motivo de vigilancia por parte de las autoridades. En consecuencia, no sólo los moralistas y decretos suntuarios se encargan de censurar libertades en el vestido y complementos, sino que estas piezas breves satirizan también su uso; basta recordar otras prendas como los sombreros de ala ancha, las lechuguillas, el guardainfante o los velos de las tapadas que serán objetos imprescindibles del paisaje urbano del XVII. Así, por ejemplo, en el anónimo Entremés de la cabellera y los muertos recogido en el Vergel de Entremeses de 1675, una mujer reclama insistentemente la presencia del alcalde porque su marido, don Quiterio, ha gastado «veinte de a ocho en una cabellera» ${ }^{15}$ que huele a cementerio. La indignación de la mujer -y con ello la comicidad - se amplía cuando descubrimos que, paradójicamente, el marido no es calvo, sino que se ha afeitado la cabeza para llevar postizo y así ir a la moda, puesto que, según afirma el propio don Quiterio: «Ninguno se la pone sin pretexto, / y en Italia, y en Francia es una cosa, / que ya todos la tienen por honrosa, / pues allá el no traerla es gran bajeza ${ }^{16}$. El postizo se manifiesta así como una marca social de prestigio desde el momento en que el no llevarla «es gran bajeza» ${ }^{17}$. De esta manera, la naturaleza del objeto establece o cimenta las relaciones sociales y se conforma como componente fundamental en la articulación de relaciones personales. Su esposa manifiesta su oposición con rotundidad al apuntar que: «Mal aya quien tal uso trajo a España» ${ }^{18}$, criticando la introducción de modas extranjeras en el país, para lo cual pide ayuda a una amiga para hacerle una burla a su marido. Así, proponen pagar a tres hombres para que hagan de muertos y asusten a don Quiterio y su criado. El entremés se resuelve con la presencia de estos 'muertos vivientes' reclamando su cabello, tras lo cual don Quiterio pronuncia su discurso moralizante:

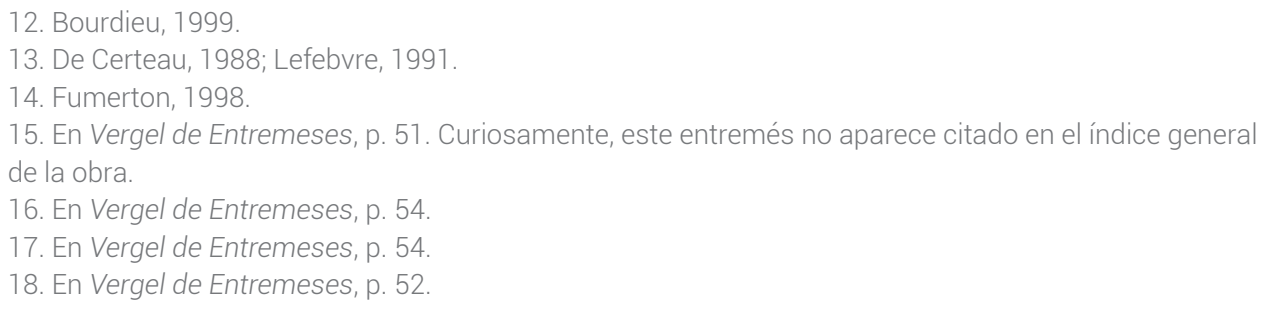




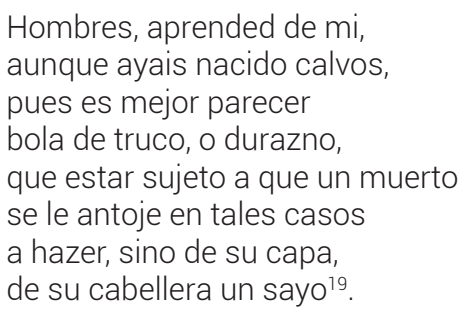

Como vemos, la cosa no va a más y la intervención de la autoridad no es necesaria debido a la presencia de los difuntos que llevan a cabo la burla e instan al marido a rechazar las nuevas modas extranjeras. Algo que no sucede en la pieza que nos ocupa a continuación. En el Entremés de Dios te la depare buena de Juan Vélez publicado en la Flor de entremeses y sainetes de 1657, la autoridad se ve burlada por su interés en abolir, mediante sentencias, los postizos, las jaulillas y demás complementos que llegan de fuera y amenazan las buenas costumbres. La pieza se abre con un alcalde enojado y amenazante que, ante el aspecto de una vecina, manifiesta:

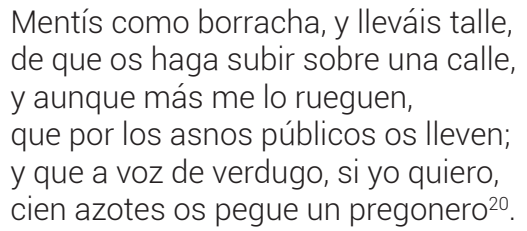

Tras escuchar los gritos, sale un Regidor y el alcalde le explica su enfado:

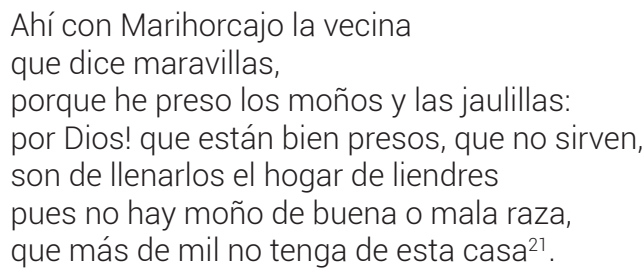

El alcalde, como medida higiénica por excusa, amenaza con quemar todos los postizos que se encuentre a su paso. La burla desemboca en demostrar la ignorancia del alcalde, quien, si bien presume de preservar las tradiciones y las buenas costumbres en su casa, - donde ni su mujer sale a dejarse ver - da pie a una muestra del rasgo ya conocido del marido cornudo:

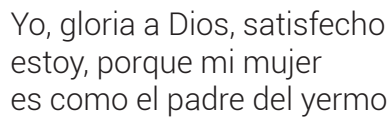

19. En Vergel de Entremeses, p. 58

20. Vélez, Entremés de Dios te la depare buena, p. 84.

21. Vélez, Entremés de Dios te la depare buena, p.84. 


\author{
que a ella no la visitan \\ sino el doctor, el barbero \\ el cura, el sacristán, \\ cuatro padres del convento, \\ el boticario y dos primos \\ suyos y el tamborilero 22 .
}

Ante tal elenco de visitas amatorias que recibe su mujer, queda patente que las nuevas modas no están reñidas con las apariencias, y no es mejor mujer aquella que sale con talle y modas cortesanas que aquella que se queda en casa preservando, en apariencia, las buenas costumbres.

Pero no todos los alcaldes se resisten a la modernidad, también hay quienes están abiertos a nuevos usos y artículos, especialmente si vienen de fuera. Es así como se puede leer en el Entremés de los enharinados recogido en Verdores del Parnaso, publicado en 1668. En esta pieza, su autor, Juan Matos Fragoso, portugués de nacimiento y madrileño de adopción, nos presenta a un barbero venido de las Indias que propone engañar a dos alcaldes mediante «infinitos remedios ${ }^{23}$ contra el crecimiento de la barba con el fin de quitarles capas y sobreros. Este ungüento indiano consiste en «unos polvillos tan fuertes / y de tan lindos compuestos / que echándolos por las barbas / del modo que yo los echo / no volverán a salir / otra vez en ningún tiempo» ${ }^{24}$. Pese a la desconfianza del Escribano, quien advierte a los alcaldes del peligro de tal negocio: "Mirad Alcaldes que juzgo / que este es un grande embustero» ${ }^{25}$, los Alcaldes deciden, no sólo contratar al Barbero para que les haga las barbas a ellos, sino a que afeite a todos los vecinos con esos polvillos que «trajo de la India hechos» ${ }^{26}$. Los nuevos objetos y materiales procedentes del Nuevo Mundo se incorporan al tejido local no sin un notable componente polémico.

El origen misterioso y exótico de la mercancía, su forma, tacto y método de aplicación desconocidos, hacen de él un objeto atractivo, algo que causa tanto admiración como miedo en una sociedad tan abierta como, en ocasiones, supersticiosa:

BARBERO Ea, aquí están ya los polvos pero mirad que os advierto que habéis de cerrar los ojos entretanto que os los echo, porque si algunos acaso os cayeren dentro de ellos son tan fuertes que bastara para que quedaseis ciegos ${ }^{27}$.

22. Vélez, Entremés de Dios te la depare buena, p. 91.

23. Matos Fragoso, Entremés de los enharinados, p. 263

24. Matos Fragoso, Entremés de los enharinados, p. 264.

25. Matos Fragoso, Entremés de los enharinados, p. 265.

26. Matos Fragoso, Entremés de los enharinados, p. 263

27. Matos Fragoso, Entremés de los enharinados, p. 267. 
Ambos alcaldes acabarán sin capas y enharinados como si estuvieran en plenas Carnestolendas. Noël Salomon ha señalado cómo el recurso del villano enharinado guardaría también relación con el miedo: «El procedimiento -afirma Salomon- viene desde hace tiempo en la historia de la farsa y, en la comedia dell'arte italiana, la blancura de la harina simboliza a menudo el miedo de un personaje» ${ }^{28}$. El problema, por tanto, no es que haya una atracción hacia lo nuevo que sea completamente inequívoca, sino que toda mención a lo desconocido o a lo que se conoce insuficientemente, arrastra consigo cierta dosis de temor o ambigüedad. $Y$ precisamente a través de esta ambigüedad que produce el elemento importado, la autoridad resulta burlada por el afán de modernidad e imitación de prácticas sociales urbanas. Para no alargarnos demasiado, sólo apuntaré una modalidad más dentro de estos 'espacios de lo cómico' que vienen determinados por los cambios de comportamiento que provocan los nuevos hábitos en la sociedad del XVII. Es el caso de las conocidas rondas de alcaldes. En ellas, generalmente, el alcalde sale a rondar acompañado del regidor dejando a su mujer en casa, a expensas del pícaro barbero, que siempre consigue convencer al marido de que entre ambos no ha pasado nada. De manera interesante, en el entremés benaventino de La ronda, el alcalde descubre a su esposa con el barbero, no en el propio hogar, sino paseando por la calle en plena noche. Al encontrarse, el representante de la autoridad no se atreverá a descubrirlos y, cuando lo haga y compruebe que la tapada es su mujer, lo más que hará será regañarla por salir de casa sin sombrero:

$\begin{array}{ll}\text { REGIDOR } & \text { ¿Es posible que a una mujer honrada } \\ & \text { y siendo de quien es, ansí venga? } \\ & \text { Tenga vergüenza. ¡Mala Pascua tenga! } \\ \text { ¿PCALDE } & \text { ¿Pues no la riñola usted sin ille nada? [...] } \\ & \text { ¿Por qué salís de casa a tales horas } \\ & \text { sin sacar un sombrero? Decí, es bueno } \\ & \text { ¿Queréis que os acatarre este sereno? }{ }^{29}\end{array}$

La pieza es de una sorprendente modernidad desde el momento en que la mujer puede salir por la noche en compañía de otro hombre que no es su marido, gracias al anonimato que ofrece la ciudad. El alcalde, por su parte, no se altera ante la situación de posible infidelidad, sino por la falta de distinción de su mujer que no lleva puesto un sombrero como es costumbre cortesana. La ropa se convierte así en algo fundamental donde los placeres visuales asumen otras connotaciones y el frenético ritmo de la moda obliga a cambiar rápidamente de un estilo a otro. Al mismo tiempo, el nuevo hábito de salir de casa por la noche y, nada menos que acompañado del amante en un síntoma de una modernidad incipiente que debe subrayarse desde el conocimiento de la novedad, desde las problemáticas de la época que muestran estas piezas y refuerzan su comicidad. Su lectura depara una reflexión sobre todo un problema moral asociado a las nuevas costumbres y, como hemos visto, a la dependencia excesiva en ciertos objetos considerados todavía 
como prescindibles en un ámbito de innovación. Los ejemplos se multiplicarían, pero en cualquier caso resulta oportuno considerar que los procesos de asimilación y diferenciación que definen estas prácticas, censurables, pero incorporadas al mundo de la ficción, ejercen un notable impacto en la producción literaria del momento. Es así cómo ciertos artículos de consumo se convierten en elementos indispensables, ya sea como rareza o como necesidad, en el cometido cómico de la autoridad en estas piezas breves, cómo el objeto se desplaza y despoja de su significado primario y se fija en el vocabulario local como una nueva resemantización mostrando el camino a una nueva sociedad de consumo, a una modernidad urbana que cristaliza a lo largo del siglo XVII.

\section{BIBLIOGRAFÍA}

Barrionuevo, Jerónimo de, Avisos de D. Jerónimo de Barrionuevo, Madrid, Tello, 1893.

Bruster, Douglas, «The New Materialism in Renaissance Studies» en Material Culture and Cultural Materialisms in the Middle Ages and the Renaissance, ed. Curtis Perry, Turnhout/Brepols, 2001, pp. 225-238.

Cáncer y Velasco, Jerónimo, Entremés de las lenguas, en Floresta de entremeses y rasgos de ocio, Madrid, Antonio de Zafra, 1691, pp. 36-45.

Certeau, Michel de, The Practice of Everyday Life, Berkeley, University of California Press, 1988.

Cotarelo y Mori, Emilio, Bibliografía de las controversias sobre la licitud del teatro en España, Madrid, Estudios de la Revista de Archivos, Bibliotecas y Museos, 1904.

Fernández Álvarez, Manuel, La sociedad española en el Siglo de Oro, Madrid, Nacional, 1983.

Fumerton, Patricia, «Introduction: A New New Historicism» en Renaissance Culture and the Everyday, ed. Patricia Fumerton y Simon Hunt, Philadelphia, University of Pennsylvania Press, 1998, pp. 1-17.

García Santo-Tomás, Enrique, Modernidad bajo sospecha. Salas Barbadillo y la cultura material del siglo XVII, Madrid, CSIC, 2008.

Lefebvre, Henri, The Production of Space, Oxford, Blackwell Publishers, 1991.

León Pinelo, Antonio, Cuestión moral si el chocolate quebranta el ayuno eclesiástico (1636), ed. Sonia Corcuera de Mancera, México, Condumex, 1994.

Madroñal Durán, Abraham, Nuevos entremeses atribuidos a Luis Quiñones de Benavente, Kassel, Edition Reichenberger, 1996.

Matos Fragoso, Juan de, Entremés de los enharinados, en Verdores del Parnaso en veinte y seis entremeses, bailes y sainetes, de diversos autores (1668), ed. Rafael Benítez Claros, Madrid, 1969, pp. 261-273. 
Ruiz Pérez, Pedro, Historia de la literatura española. El siglo del arte nuevo. 15981691, Barcelona, Crítica, 2010.

Salomon, N., Lo villano en el teatro del Siglo de Oro, Madrid, Castalia, 1985.

Valverde Turices, Santiago de, Un discurso del chocolate, Sevilla, Cabrera, 1624.

Vélez, Juan, «Entremés de Dios te la depare buena» en Flor de entremeses y sainetes de diferentes autores, Madrid, Fortanet, 1903 [1657], pp. 84-96.

Vergel de entremeses y conceptos del donaire, con diferentes bailes, loas y mojigangas. Compuesto por los mejores ingenios destos tiempos (1675), ed. Jesús Cañedo Fernández, Madrid, Biblioteca de Antiguos Libros Hispánicos, 1970, pp. 51-60. 